\begin{tabular}{|c|l|}
\hline Title & $\begin{array}{l}\text { Synthesis of pinacol aryl boronates via cross coupling reaction of bis(pinacolato)diboron with chloroarenes catalyzed by } \\
\text { pall ladium(0)-tricyclohexy lphosphine complexes }\end{array}$ \\
\hline Author(s) & Ishiyama, Tatsuo; Ishida, Kousaku; Miy aura, Norio \\
\hline Citation & $\begin{array}{l}\text { Tetrahedron, 57(49), 9813-9816 } \\
\text { https://doi.org/10.1016/50040-4020(01)00998 X }\end{array}$ \\
\hline Issue Date & 2001-12-03 \\
\hline Doc URL & http://hdl.handle.net/2115/15838 \\
\hline Type & article (author version) \\
\hline File Information & TETR57-49.pdf \\
\hline
\end{tabular}

Instructions for use 


\title{
Synthesis of Pinacol Arylboronates via Cross-Coupling Reaction of \\ Bis(pinacolato)diboron with Chloroarenes Catalyzed by \\ Palladium(0)-Tricyclohexylphosphine Complexes
}

\author{
Tatsuo Ishiyama*, Kousaku Ishida, and Norio Miyaura* \\ Division of Molecular Chemistry, Graduate School of Engineering, \\ Hokkaido University, Sapporo 060-8628, Japan
}

\begin{abstract}
The cross-coupling reaction of bis(pinacolato)diboron with chloroarenes to yield pinacol arylboronates was carried out in 1,4-dioxane at $80{ }^{\circ} \mathrm{C}$ in the presence of KOAc (1.5 equivs) and $\mathrm{Pd}(\mathrm{dba})_{2} / 2.4 \mathrm{PC}_{3}(3-6 \mathrm{~mol} \%)$. The catalyst was also effective to carry out analogous coupling with aryl bromides or triflates under milder conditions than those of the previous procedures catalyzed by $\mathrm{PdCl}_{2}(\mathrm{dppf})$ in DMSO.

Keywords: Boron and compounds; Coupling reactions; Aryl halides; Palladium and compounds
\end{abstract}

\section{Introduction}


The cross-coupling reaction between tetra(alkoxo)diborons and aryl halides or triflates in the presence of a base and $\mathrm{PdCl}_{2}(\mathrm{dppf})$ provides a convenient and direct method for the synthesis of arylboronates from aryl electrophiles. ${ }^{1}$ The coupling reaction is feasible with various functional groups, e.g., $\mathrm{CO}_{2} \mathrm{Me}, \mathrm{COMe}, \mathrm{NO}_{2}$, and $\mathrm{CN}$, which need protection-deprotection in the two-step procedure for preparing arylboronic acids or esters from magnesium or lithium reagents. ${ }^{2}$ The protocol offers a direct and efficient route to variously functionalized boronic esters, the utility of which has been amply demonstrated in the synthesis of natural products, ${ }^{3}$ biologically active compounds, ${ }^{4}$ and functional materials. ${ }^{5}$ On the other hand, the direct preparation of arylboronic esters from aryl halides or triflates now allows a one-pot, two-step procedure ${ }^{1 \mathrm{~b}, 6}$ and a solid-phase method ${ }^{7}$ for the synthesis of unsymmetrical biaryls. The cross-coupling reaction of pinacolborane with haloarenes in the presence of a palladium catalyst and triethylamine reported by Masuda and Murata is another convenient and economical variant for the preparation of such pinacol arylboronates. ${ }^{8}$

In this paper, we report the palladium-catalyzed coupling reaction of chloroarenes (1) with bis(pinacolato)diboron (2) for the synthesis of pinacol arylboronates (3) (Scheme 1). Chloroarene is a desirable substrate for the cross-coupling reactions in combinatorial synthesis since a large number of derivatives is commercially available. Although their oxidative addition to palladium(0) complexes is very slow, a catalyst in situ generated from $\mathrm{Pd}(\mathrm{dba})_{2}$ and $\mathrm{PCy}_{3}$ efficiently catalyzed the reaction at $80{ }^{\circ} \mathrm{C}$. The catalyst can be also effective to carry out the coupling reaction of less reactive aryl bromides or triflates 
bearing electron-donating substituents under mild conditions.

$<<$ Scheme $1>>$

\section{Results and Discussion}

\section{Reaction conditions}

Various phosphine ligands are effective in stabilizing the palladium(0) species; however, the stoichiometry of phosphine to palladium and the bulkiness ${ }^{9}$ or donating ability ${ }^{9,10}$ of phosphine ligands change the reactivity of catalysts toward oxidative addition and transmetalation. ${ }^{11}$ Palladium complexes having fewer than four phosphines, a weakly coordinating ligand such as $\mathrm{AsPh}_{3},{ }^{12}$ or a bulky phosphine such as $\mathrm{P}(\mathrm{o} \text {-tolyl })_{3},{ }^{13}$ provide a highly reactive catalyst because of the ready formation of the coordinatively unsaturated species. Another role of the ligand is electron donation to the palladium(0) metal center, which was amply demonstrated in the cross-coupling reaction of chloroarenes. Bulky and highly donating ligands, such as $\mathrm{P}(t-\mathrm{Bu})_{3},{ }^{14}$ $o$-(di-t-butylphosphino)biphenyl (5), ${ }^{15}$ and $N$-heterocyclic carbenes, ${ }^{16}$ were recognized to be highly effective for chloroarenes even at room temperature. The high reactivity of the complexes involving these ligands is attributable to the strong electron-donating ability of the ligand to the metal center and the ready dissociation of the ligand to generate a coordinatively unsaturated species. It was also noted that less bulky phosphines, such as (dicyclohexylphosphino)arenes (e.g., $\quad 7)^{17}$ and tricyclohexylphosphine $\left(\mathrm{PCy}_{3}\right),{ }^{14,18}$ are a practical ligand giving a more stable complex 
at high temperature.

A screening of representative phosphine ligands reported in the cross-coupling reactions of chloroarenes is summarized in Table 1 . The catalysts were in situ generated by stirring a mixture of $\mathrm{Pd}(\mathrm{dba})_{2}(3 \mathrm{~mol} \%)$ and a phosphine ligand (3.3-7.2 mol\%) at room temperature to be used directly for the coupling reaction of diboron (2) (1.1 equivs) and 4-chlorobenzaldehyde (1.0 equiv) in the presence of KOAc (1.5 equivs) in dioxane. Use of triarylphosphines commonly resulted in low conversions and low yields due to their low electron-donating ability (entries 2-5), whereas more electron-rich tris(p-methoxyphenyl)phosphine exhibited better catalyst activity than triphenylphosphine (entries 2 and 4). Among the four phosphine ligands developed by Buchwald and Guram (4-7), 4, 5, and 7 achieved high yields in a range of $68-78 \%$ (entries 6-9). However, a combination of $\mathrm{Pd}(\mathrm{dba})_{2}$ and $\mathrm{PCy}_{3}$ (2.4 equivs) was finally recognized to be the most efficient catalyst to achieve a quantitative conversion and an almost quantitative yield at $80{ }^{\circ} \mathrm{C}$ (entry 10$)$. On the other hand, the palladium-P(t-Bu $)_{3}$ complex resulted in low yields at temperatures in the range of $20^{\circ} \mathrm{C}$ to $80^{\circ} \mathrm{C}$ (entry 11 ). $<<$ Table $1>>$

KOAc was found to be the best base for the coupling of diboron (2) with bromo- or iodoarenes because a stronger base, such as $\mathrm{K}_{3} \mathrm{PO}_{4}$ and $\mathrm{K}_{2} \mathrm{CO}_{3}$, prompted the further coupling of arylboronates (3) with haloarenes, resulting in a competitive formation of homo-coupling biaryls (36-60\% yields). The use of KOAc was also significant in the present coupling reaction catalyzed by a palladium- $\mathrm{PCy}_{3}$ complex. The reaction was 
almost quantitative with KOAc (94\%), whereas use of $\mathrm{K}_{2} \mathrm{CO}_{3}, \mathrm{~K}_{3} \mathrm{PO}_{4}$, and $\mathrm{KOPh}$ resulted in $85 \%, 75 \%$, and 51\% yields respectively.

\section{Scope and limitation}

The borylation of the representative chloroarenes is summarized in Table 2. The reaction was very fast, completing within $6 \mathrm{~h}$ for activated chloroarenes possessing an electron-withdrawing group such as $\mathrm{NO}_{2}, \mathrm{CN}, \mathrm{CHO}$, and $\mathrm{CO}_{2} \mathrm{Me}$ (entries 1-6). However, the presence of ortho-substituents (entries 2 and 4) or electron-donating substituents (entries 8-13) slowed down the reaction significantly, thus requiring prolongation of the time to $48 \mathrm{~h}$ or a higher catalyst loading (6 mol\%) to complete the reaction. Analogously to the previous borylation of aryl bromides, iodides, and triflates, the reaction tolerated various functional groups. The reaction is applicable for the borylation of heteroaromatic chlorides such as 3-chloropyridine (entry 16), 2-chlorobenzo[b]furan (entry 17), 4-chloroindole (entry 18), and presumably other derivatives reported previously; however, 2-chloropyridine failed to yield the corresponding boronate (entry 15). Since the boronic acids and esters adjacent to a heteroatom are highly susceptible to hydrolytic protodeboronation, ${ }^{19}$ the coupling reaction can be often followed by hydrolytic boron-carbon bond cleavage. Although the reaction in dioxane can be much more insensitive to such protodeboronation than that in DMSO, the 2-borylpyridine was quantitatively led to free pyridine. Such side-reaction giving the reduction product of haloarenes in small amounts was also observed in the reactions of 2-chloronitrobenzene and 2-chlorobenzonitrile (entries 2 and 4). 
$<<$ Table 2>>

The use of such highly electron-donating ligand $\left(\mathrm{PCy}_{3}\right)$ was also advantageous to significantly shorten the reaction times of the borylation of electron-rich aryl bromides or triflates (Scheme 2). The borylation of iodoarenes is fast under the previous conditions using $\mathrm{PdCl}_{2}(\mathrm{dppf})$ in DMSO, even for a dimethylamino derivative; however, analogous reactions of the bromides or triflates are significantly slow. In contrast to the palladium-dppf complex, $\mathrm{Pd}(\mathrm{dba})_{2} / \mathrm{PCy}_{3}$ completed the reactions of both 4-methoxy and 4-dimethylamino derivatives within $7 \mathrm{~h}$. Although the efficiency of $\mathrm{Pd}(\mathrm{dba})_{2} / \mathrm{PCy}_{3}$ has not yet been studied in detail for other halides or triflates, the catalyst would serve to achieve complete conversions within shorter reaction times than those of the previously reported procedure using $\mathrm{PdCl}_{2}(\mathrm{dppf})$.

$<<$ Scheme 2>>

\section{Experimental}

\section{Reagents}

$\operatorname{Pd}(\mathrm{dba})_{2}{ }^{20}$ and bis(pinacolato)diboron ${ }^{21}$ were synthesized by the reported procedures. All phosphine ligands purchased from Strem were used directly without further purification. 1,4-Dioxane was distilled from sodium and KOAc was dried in vacuo.

\section{Representative procedure (Table 2)}


A 25 ml-flask assembled a magnetic stirring bar, a septum inlet, and a condenser was charged with $\mathrm{Pd}(\mathrm{dba})_{2}(0.017 \mathrm{~g}, 0.03 \mathrm{mmol}, 3 \mathrm{~mol} \%)$ and tricyclohexylphosphine $\left(\mathrm{PCy}_{3}\right.$, $0.020 \mathrm{~g}, 0.072 \mathrm{mmol})$, and flushed with nitrogen. Dioxane $(6 \mathrm{ml})$ was added and the resulting mixture was then stirred for $30 \mathrm{~min}$ at room temperature. Bis(pinacolato)diboron (2, $0.279 \mathrm{~g}, 1.1 \mathrm{mmol})$, KOAc (0.147 g, $1.5 \mathrm{mmol})$, and a chloroarene $(1.0 \mathrm{mmol})$ were added successively. After being stirred at $80{ }^{\circ} \mathrm{C}$ for the period shown in Table 2, the reaction mixture was treated with water $(5 \mathrm{ml})$ at room temperature. The product was extracted with benzene, washed with brine, and dried over $\mathrm{MgSO}_{4}$. Kugelrohr distillation gave an analytically pure sample.

The following arylboronates were prepared by the above general procedure, unless otherwise noted.

2-(4,4,5,5-Tetramethyl-1,3,2-dioxaborolan-2-yl)benzonitrile (entry 4). ${ }^{1} \mathrm{H}$ NMR (400 MHz, $\left.\mathrm{CDCl}_{3}, \mathrm{TMS}\right) \delta 1.39$ (s, 12H), 7.52 (dt, $\left.J=1.5,7.4 \mathrm{~Hz}, 1 \mathrm{H}\right), 7.57$ (dt, $J=1.5,7.4$ $\mathrm{Hz}, 1 \mathrm{H}), 7.70$ (dd, $J=1.5,7.3 \mathrm{~Hz}, 1 \mathrm{H}), 7.88$ (d, $J=7.3 \mathrm{~Hz}, 1 \mathrm{H}) ;{ }^{13} \mathrm{C}$ NMR (100 MHz, $\mathrm{CDCl}_{3}$, TMS) $\delta 24.77,84.77,117.24,118.96,131.06,131.54,133.40,135.82 ;{ }^{11} \mathrm{~B}$ NMR $\left(128 \mathrm{MHz}, \mathrm{CDCl}_{3}, \mathrm{BF}_{3} \cdot \mathrm{OEt}_{2}\right) \delta$ 30.74; exact mass calcd for $\mathrm{C}_{13} \mathrm{H}_{16} \mathrm{BNO}_{2}$ 229.1274, found 229.1270.

3-(4,4,5,5-Tetramethyl-1,3,2-dioxaborolan-2-yl)anisole (entry 11). ${ }^{1} \mathrm{H}$ NMR (400 MHz, $\left.\mathrm{CDCl}_{3}, \mathrm{TMS}\right) \delta 1.35$ (s, 12H), 3.84 (s, 3H), 7.01 (dd, J=2.7, $\left.8.1 \mathrm{~Hz}, 1 \mathrm{H}\right), 7.30$ (t, 
$J=7.8 \mathrm{~Hz}, 1 \mathrm{H}), 7.33$ (d, $J=2.7 \mathrm{~Hz}, 1 \mathrm{H}), 7.40$ (d, $J=7.1 \mathrm{~Hz}, 1 \mathrm{H}) ;{ }^{13} \mathrm{C}$ NMR $(100 \mathrm{MHz}$, $\left.\mathrm{CDCl}_{3}, \mathrm{TMS}\right) \delta 24.85,55.24,83.82,117.91,118.64,127.17,128.93,159.02 ;{ }^{11} \mathrm{~B} \mathrm{NMR}$ (128 MHz, $\mathrm{CDCl}_{3}, \mathrm{BF}_{3} \cdot \mathrm{OEt}_{2}$ ) $\delta$ 30.56; exact mass calcd for $\mathrm{C}_{13} \mathrm{H}_{19} \mathrm{BO}_{3}$ 234.1427, found 234.1432.

3-(4,4,5,5-Tetramethyl-1,3,2-dioxaborolan-2-yl)pyridine (entry 16). ${ }^{1} \mathrm{H}$ NMR (400 MHz, $\left.\mathrm{CDCl}_{3}, \mathrm{TMS}\right) \delta 1.36$ (s, 12H), 7.28 (ddd, $J=0.9,4.9,7.6 \mathrm{~Hz}, 1 \mathrm{H}$ ), 8.05 (dt, $J=7.6$, $1.8 \mathrm{~Hz}, 1 \mathrm{H}), 8.67$ (dd, $J=2.0,4.9 \mathrm{~Hz}, 1 \mathrm{H}), 8.95$ (s, $1 \mathrm{H}) ;{ }^{13} \mathrm{C}$ NMR $\left(100 \mathrm{MHz}, \mathrm{CDCl}_{3}\right.$, TMS) $\delta$ 24.84, 84.20, 123.05, 142.18, 151.98, 155.48; ${ }^{11} \mathrm{~B}$ NMR (128 MHz, $\mathrm{CDCl}_{3}$, $\left.\mathrm{BF}_{3} \cdot \mathrm{OEt}_{2}\right) \delta$ 31.00; exact mass calcd for $\mathrm{C}_{11} \mathrm{H}_{16} \mathrm{BNO}_{2}$ 205.1274, found 205.1281.

2-(4,4,5,5-Tetramethyl-1,3,2-dioxaborolan-2-yl)benzo[b]furan (entry 17). ${ }^{1} \mathrm{H}$ NMR (400 MHz, $\left.\mathrm{CDCl}_{3}, \mathrm{TMS}\right) \delta 1.39$ (s, 12H), 7.23 (t, $\left.J=7.4 \mathrm{~Hz}, 1 \mathrm{H}\right), 7.34$ (dt, $J=1.2,7.8 \mathrm{~Hz}$, 1H), 7.40 (s, 1H), 7.57 (d, J=8.5 Hz, 1H), 7.63 (d, $J=7.8 \mathrm{~Hz}, 1 \mathrm{H}) ;{ }^{13} \mathrm{C}$ NMR (100 MHz, $\left.\mathrm{CDCl}_{3}, \mathrm{TMS}\right) \delta$ 24.77, 84.68, 111.97, 119.53, 121.88, 122.71, 125.93, 127.48, 157.51;

${ }^{11} \mathrm{~B}$ NMR (128 $\left.\mathrm{MHz} \mathrm{CDCl}_{3}, \mathrm{BF}_{3} \bullet \mathrm{OEt}_{2}\right) \delta 27.51$; exact mass calcd for $\mathrm{C}_{14} \mathrm{H}_{17} \mathrm{BO}_{3}$ 244.1270, found 244.1264.

4-(4,4,5,5-Tetramethyl-1,3,2-dioxaborolan-2-yl)indole (entry 18). ${ }^{1} \mathrm{H}$ NMR (400 MHz, $\left.\mathrm{CDCl}_{3}, \mathrm{TMS}\right) \delta 1.39$ (s, 12H), 7.06 (ddd, $\left.J=1.0,2.1,3.0 \mathrm{~Hz}, 1 \mathrm{H}\right), 7.21$ (dd, $J=7.1$, $8.1 \mathrm{~Hz}, 1 \mathrm{H}), 7.26$ (t, $J=2.8 \mathrm{~Hz}, 1 \mathrm{H}), 7.50$ (d, $J=8.1 \mathrm{~Hz}, 1 \mathrm{H}), 7.64$ (dd, $J=0.7,7.1 \mathrm{~Hz}$, 1H), 8.17 (br s, $1 \mathrm{H}) ;{ }^{13} \mathrm{C}$ NMR (100 MHz, $\mathrm{CDCl}_{3}$, TMS) $\delta$ 24.97, 83.38, 104.50, 113.96, 
121.30, 124.58, 127.84, 132.49, 135.09; ${ }^{11} \mathrm{~B} \mathrm{NMR}\left(128 \mathrm{MHz}, \mathrm{CDCl}_{3}, \mathrm{BF}_{3} \cdot \mathrm{OEt}_{2}\right) \delta$ 31.05; exact mass calcd for $\mathrm{C}_{14} \mathrm{H}_{18} \mathrm{BNO}_{2} 243.1430$, found 243.1422 .

Syntheses of 4-(4,4,5,5-tetramethyl-1,3,2-dioxaborolan-2-yl)nitrobenzene (entry 1), ${ }^{1,8}$ 2-(4,4,5,5-tetramethyl-1,3,2-dioxaborolan-2-yl)nitrobenzene (entry $\quad 2){ }^{1}$ 4-(4,4,5,5-tetramethyl-1,3,2-dioxaborolan-2-yl)benzonitrile $\quad$ (entry $\quad 3),{ }^{1,8}$ 4-(4,4,5,5-tetramethyl-1,3,2-dioxaborolan-2-yl)benzaldehyde $\quad($ entry $\quad 5),{ }^{1} \quad$ methyl 4-(4,4,5,5-tetramethyl-1,3,2-dioxaborolan-2-yl)benzoate (entry $6),{ }^{1}$ (4,4,5,5-tetramethyl-1,3,2-dioxaborolan-2-yl)benzene (entry 7), ${ }^{1,8}$ 4-(4,4,5,5-tetramethyl-1,3,2-dioxaborolan-2-yl)toluene (entry 8), ${ }^{8}$ 2-(4,4,5,5-tetramethyl-1,3,2-dioxaborolan-2-yl)toluene (entry

$9), 8$ 4-(4,4,5,5-tetramethyl-1,3,2-dioxaborolan-2-yl)anisole (entry $10),{ }^{1,8}$ 2-(4,4,5,5-tetramethyl-1,3,2-dioxaborolan-2-yl)anisole (entry $12){ }^{1}$ $N, N$-dimethyl-4-(4,4,5,5-tetramethyl-1,3,2-dioxaborolan-2-yl)aniline (entry 13), ${ }^{1,8}$ and 2-(4,4,5,5-tetramethyl-1,3,2-dioxaborolan-2-yl)naphthalene (entry 14$)^{8}$ were previously reported.

\section{References}

1. a) For a review: Ishiyama, T.; Miyaura, N. J. Organomet. Chem. 2000, 611, 392. b) Ishiyama, T.; Itoh, Y.; Kitano, T.; Miyaura, N. Tetrahedron Lett. 1997, 38, 3447. c) Ishiyama, T.; Murata, M.; Miyaura, N. J. Org. Chem. 1995, 60, 7508. 
2. a) Onak, T. Organoborane Chemistry; Academic: New York, 1975. b) Nesmeyanov, A. N.; Sokolik, R. A. Methods of Elemento-Organic Chemistry; North-Holland: Amsterdam, 1967; Vol. 1.

3. a) Elder, A. M.; Rich, D. H. Org. Lett. 1999, 1, 1443. b) Jung, M. E.; Lazarova, T. I. J. Org. Chem. 1999, 64, 2976. c) Zembower, D. E.; Zhang, H. J. Org. Chem. 1998, 63, 9300. d) Brimble, M. A.; Duncalf, L. J.; Neville, D. J. Chem. Soc., Perkin Trans. 1 1998, 4165.

4. a) McKenna, J. M.; Moliterni, J.; Qiao, Y. Tetrahedron Lett. 2001, 42, 5797. b) Carbonnelle, A.-C.; Zhu, J. Org. Lett. 2000, 2, 3477. c) Firooznia, F.; Gude, C.; Chan, K.; Marcopulos, N.; Satoh, Y. Tetrahedron Lett. 1999, 40, 213. d) Malan, C.; Morin, C. J. Org. Chem. 1998, 63, 8019. e) Nakamura, H.; Fujiwara, M.; Yamamoto, Y. J. Org. Chem. 1998, 63, 7529.

5. a) Sakai, N.; Gerard, D.; Matile, S. J. Am. Chem. Soc. 2001, 123, 2517. b) Iovine, P. M.; Kellett, M. A.; Redmore, N. P.; Therien, M. J. J. Am. Chem. Soc. 2000, 122, 8717. c) Yu, H.-B.; Hu, Q.-S.; Pu, L. J. Am. Chem. Soc. 2000, 122, 6500. d) Wang, S.; Oldham, W. J., Jr.; Hudack, R. A., Jr.; Bazan, G. C. J. Am. Chem. Soc. 2000, 122, 5695. e) Deng, Y.; Chang, C. K.; Nocera, D. G. Angew. Chem. Int. Ed. 2000, 39, 1066.

6. Giroux, A.; Han, Y.; Prasit, P. Tetrahedron Lett. 1997, 38, 3841.

7. a) Gosselin, F.; Betsbrugge, J. V.; Hatam, M.; Lubell, W. D. J. Org. Chem. 1999, 64, 2486. b) Tempest, P. A.; Armstrong, R. W. J. Am. Chem. Soc. 1997, 119, 7607. c) Piettre, S. R.; Baltzer, S. Tetrahedron Lett. 1997, 38, 1197. d) Brown, S. D.; Armstrong, R. W. J. Am. Chem. Soc. 1996, 118, 6331. 
8. a) Murata, M.; Oyama, T.; Watanabe, S.; Masuda, Y. J. Org. Chem. 2000, 65, 164.

b) Murata, M.; Watanabe, S.; Masuda, Y. J. Org. Chem. 1997, 62, 6458.

9. Tolman, C. A. Chem. Rev. 1977, 77, 313 and references therein

10. Fernandez, A. L.; Reyes, C.; Prock, A.; Giering, W. P. J. Chem. Soc., Perkin Trans. 2, 2000, 1033 and references therein.

11. For Reviews: a) Suzuki, A. In Metal-Catalyzed Cross-Coupling Reactions; Diederich, F., Stang, P. J. Eds.; Wiley-VCH: Weinheim, 1998; p 49. b) Miyaura, N. In Advances in Metal-Organic Chemistry; Liebeskind, L. S. Ed.; JAI: London, 1998; Vol. 6, p 187. c) Miyaura, N.; Suzuki, A. Chem. Rev. 1995, 95, 2457.

12. a) Kojima, A.; Honzawa, S.; Boden, C. D. J.; Shibasaki, M. Tetrahedron Lett. 1997, 38, 3455. b) Ahiko, T.-a.; Ishiyama, T.; Miyaura, N. Chem. Lett. 1997, 811.

13. a) Boger, D. L.; Miyazaki, S.; Kim, S. H.; Wu, J. H.; Castle, S. L.; Loiseleur, O.; Jin, Q. J. Am. Chem. Soc. 1999, 121, 10004. b) Hensel, V.; Lützow, K.; Jacob, J.; Gessler, K.; Saenger, W.; Schlüter, A. D. Angew. Chem. Int. Ed. Engl. 1997, 36, 2654.

14. a) Littke, A. F.; Dai, C.; Fu, G. C. J. Am. Chem. Soc. 2000, 122, 4020. b) Littke, A. F.; Fu, G. C. Angew. Chem. Int. Ed. 1998, 37, 3387.

15. a) Wolfe, J. P.; Singer, R. A.; Yang, B. H.; Buchwald, S. L. J. Am. Chem. Soc. 1999, 121, 9550. b) Wolfe, J. P.; Buchwald, S. L. Angew. Chem. Int. Ed. 1999, 38, 2413.

16. a) Zhang, C.; Trudell, M. L. Tetrahedron Lett. 2000, 41, 595. b) Zhang, C.; Huang, J.; Trudell, M. L.; Nolan, S. P. J. Org. Chem. 1999, 64, 3804.

17. a) Bei, X.; Turner, H. W.; Weinberg, W. H.; Guram, A. S. J. Org. Chem. 1999, 64, 6797. b) Bei, X.; Crevier, T.; Guram, A. S.; Jandeleit, B.; Powers, T. S.; Turner, H. W.; 
Uno, T.; Weinberg, W. H. Tetrahedron Lett. 1999, 40, 3855.

18. Shen, W. Tetrahedron Lett. 1997, 38, 5575.

19. Abraham, M. H.; Grellier, P. L. In The Chemistry of the Metal-Carbon Bond; Hartley, F. R.; Patai, S., Eds.; Wiley: New York, 1985, Vol. 2, p 25.

20. Ukai, T.; Kawazura, H.; Ishii, Y. J. Organomet. Chem. 1974, 65, 253.

21. Ishiyama, T.; Murata, M.; Ahiko, T.-a.; Miyaura, N. Organic Syntheses 2000, 77, 176. 


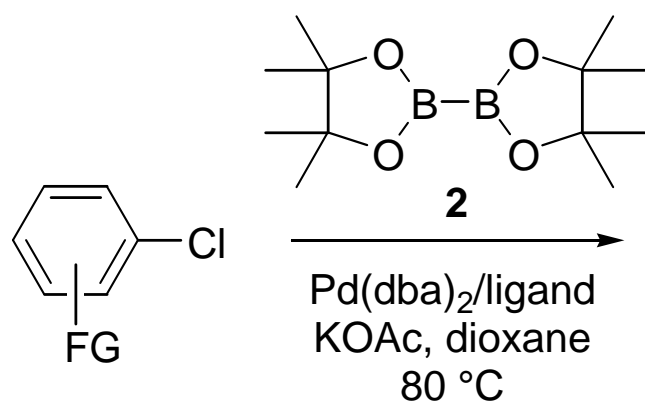

1

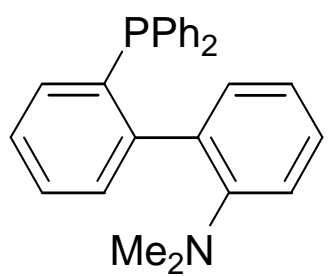

4<smiles>CC1(c2ccccc2P)OCCO1</smiles>

6

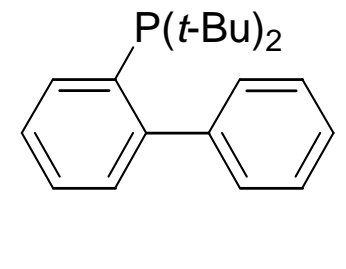

5

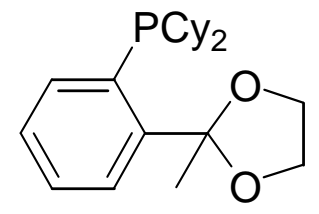

7<smiles>CC1(C)OB(c2ccccc2)OC1(C)C</smiles>

3<smiles>CC(=P)C1CCCCCC1</smiles>

$\mathrm{PCy}_{3}$<smiles>CC(C)(C)C(C)(C)P</smiles>

$\mathrm{P}(t-\mathrm{Bu})_{3}$

Scheme 1. Synthesis of pinacol arylboronates via cross-coupling reaction of diboron with chloroarenes. 
Table 1. Effect of ligands in the reaction of bis(pinacolato)diboron (2, 1.1 mmol) with 4-chlorobenzaldehyde $(1.0 \mathrm{mmol})$ in dioxane $(6 \mathrm{ml})$ at $80{ }^{\circ} \mathrm{C}$ for $16 \mathrm{~h}$ in the presence of $\mathrm{KOAc}(1.5 \mathrm{mmol}), \mathrm{Pd}(\mathrm{dba})_{2}$ (3 mol\%), and a phosphine ligand (3.3-7.2 mol\%)

\begin{tabular}{clcc}
\hline entry & ligand & ligand/equivs $^{a}$ & yield/\% ${ }^{b}$ \\
\hline 1 & none & & 1 \\
2 & $\mathrm{PPh}_{3}$ & 2.4 & 19 \\
3 & $\left.\mathrm{P}^{\circ}-\mathrm{MeC}_{6} \mathrm{H}_{4}\right)_{3}$ & 2.4 & 36 \\
4 & $\mathrm{P}\left(p-\mathrm{MeOC}_{6} \mathrm{H}_{4}\right)_{3}$ & 2.4 & 44 \\
5 & $\mathrm{dppf}^{c}$ & 1.1 & 18 \\
6 & $\mathbf{4}$ & 1.1 & 78 \\
7 & $\mathbf{5}$ & 2.4 & 68 \\
8 & $\mathbf{6}$ & 1.1 & 10 \\
9 & $\mathbf{7}$ & 1.1 & 71 \\
10 & $\mathrm{PC} y_{3}$ & 2.4 & 94 \\
11 & $\mathrm{P}(t-\mathrm{Bu})_{3}$ & 2.4 & 58 \\
\hline
\end{tabular}

${ }^{a}$ Equivalents to palladium metal.

${ }^{b} \mathrm{GC}$ yields based on 4-chlorobenzaldehyde.

${ }^{c} 1,1$ '-bis(diphenylphosphino)ferrocene. 
Table 2. Synthesis of pinacol arylboronates

\begin{tabular}{|c|c|c|c|c|}
\hline entry & chloroarene & $\operatorname{mol} \%^{a}$ & time/h & yield $/ \%^{b}$ \\
\hline 1 & $4-\mathrm{O}_{2} \mathrm{NC}_{6} \mathrm{H}_{4} \mathrm{Cl}$ & 3 & 4 & 88 \\
\hline 2 & $2-\mathrm{O}_{2} \mathrm{NC}_{6} \mathrm{H}_{4} \mathrm{Cl}$ & 3 & 48 & 72 \\
\hline 3 & $4-\mathrm{NCC}_{6} \mathrm{H}_{4} \mathrm{Cl}$ & 3 & 4 & 98 \\
\hline 4 & $2-\mathrm{NCC}_{6} \mathrm{H}_{4} \mathrm{Cl}$ & 3 & 48 & 72 \\
\hline 5 & $4-\mathrm{OHCC}_{6} \mathrm{H}_{4} \mathrm{Cl}$ & 3 & 6 & 94 \\
\hline 6 & $4-\mathrm{MeO}_{2} \mathrm{CC}_{6} \mathrm{H}_{4} \mathrm{Cl}$ & 3 & 6 & 87 \\
\hline 7 & $\mathrm{C}_{6} \mathrm{H}_{5} \mathrm{Cl}$ & 6 & 48 & 91 \\
\hline 8 & $4-\mathrm{MeC}_{6} \mathrm{H}_{4} \mathrm{Cl}$ & 6 & 48 & 92 \\
\hline 9 & $2-\mathrm{MeC}_{6} \mathrm{H}_{4} \mathrm{Cl}$ & 3 & 48 & 86 \\
\hline 10 & $4-\mathrm{MeOC}_{6} \mathrm{H}_{4} \mathrm{Cl}$ & 6 & 48 & 78 \\
\hline 11 & $3-\mathrm{MeOC}_{6} \mathrm{H}_{4} \mathrm{Cl}$ & 6 & 48 & 92 \\
\hline 12 & $2-\mathrm{MeOC}_{6} \mathrm{H}_{4} \mathrm{Cl}$ & 3 & 48 & 70 \\
\hline 13 & $4-\mathrm{Me}_{2} \mathrm{NC}_{6} \mathrm{H}_{4} \mathrm{Cl}$ & 6 & 48 & 73 \\
\hline 14 & 2-chloronaphthalene & 3 & 48 & 77 \\
\hline 15 & 2-chloropyridine & 3 & 48 & 0 \\
\hline 16 & 3-chloropyridine & 3 & 48 & 82 \\
\hline 17 & & 3 & 48 & 73 \\
\hline 18 & $-\mathrm{Cl}$ & 3 & 48 & 72 \\
\hline
\end{tabular}

${ }^{a} \mathrm{Mol} \%$ of catalyst.

${ }^{b} \mathrm{GC}$ yields based on chloroarenes. 


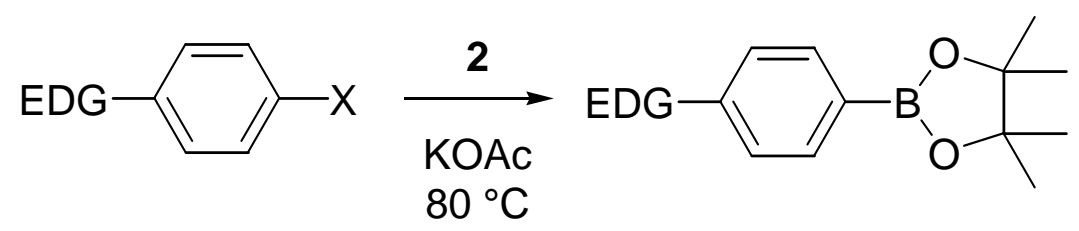

EDG $\quad \mathrm{X}=$ catalyst/solvent $\quad$ time/h yield/\%

$\begin{array}{lllcl}\mathrm{MeO} & \mathrm{I} & \mathrm{PdCl}_{2}(\mathrm{dppf}) / \mathrm{DMSO} & 2 & 82 \\ & \mathrm{Br} & \mathrm{PdCl}_{2}(\mathrm{dppf}) / \mathrm{DMSO} & 24 & 69 \\ & \mathrm{Br} & \mathrm{Pd}(\mathrm{dba})_{2}-\mathrm{PCy} / \text { dioxane } & 7 & 81 \\ & \mathrm{OTf} & \mathrm{PdCl}_{2}(\mathrm{dppf})-\mathrm{dppf} / \text { dioxane } & 13 & 93 \\ & \mathrm{OTf} & \mathrm{Pd}(\mathrm{dba})_{2}-\mathrm{PCy} / \text { dioxane } & 2 & 83 \\ \mathrm{Me}_{2} \mathrm{~N} & \mathrm{I} & \mathrm{PdCl}_{2}(\mathrm{dppf}) / \mathrm{DMSO} & 6 & 90 \\ & \mathrm{Br} & \mathrm{PdCl}_{2}(\mathrm{dppf}) / \mathrm{DMSO} & 24 & 23 \\ & \mathrm{Br} & \mathrm{Pd}(\mathrm{dba})_{2}-\mathrm{PC} \mathrm{y}_{3} / \mathrm{dioxane} & 6 & 81\end{array}$

Scheme 2. Cross-coupling reaction of diboron with electron-rich aryl electrophiles. 